\title{
Surgical Correction of Congenital Lobar Emphysema in a Dog
}

\author{
Hideki MATSUMOTO ${ }^{1,2)}$, Takeshi KAKEHATA ${ }^{3)}$, Takefumi HYODO $^{4)}$, Kensei HANADA ${ }^{5)}$, Yoko TSUJ ${ }^{3)}$, \\ Satoshi HOSHINO ${ }^{6}$ and Hiroshi ISOMURA ${ }^{7)}$
}

\begin{abstract}
${ }^{1)}$ Animal Clinical Research Foundation, 214-10 Yatsuya, Kurayoshi-shi, Tottori 682-0025, 2)Matsumoto Animal Hospital, 2-35-6 Kikyocho, Hakodate-shi, Hokkaido 041-0808, ${ }^{3)}$ Kakehata Veterinary Hospital, 2-14-21 Nanaehama, Kamiiso-cho, Kamiiso-gun, Hokkaido 049-0111, ${ }^{4}$ Hyoudo Animal Hospital, 1-5 Kaigan-cho, Hakodate-shi, Hokkaido 040-0061, ${ }^{5}$ Showa Animal Hospital, 1-17 Fukaboricho, Hakodate-shi, Hokkaido 042-0941, ${ }^{6)}$ Hoshino Veterinary Hospital, N-40,E-5-1-1, Higashi-ku, Sapporo, Hokkaido 007-0840 and ${ }^{7}$ Sapporo General Pathology Laboratory Co., Ltd., S-12, W-18-3-17, Chuo-ku, Sapporo, Hokkaido 064-0912, Japan
\end{abstract}

(Received 4 April 2003/Accepted 14 October 2003)

ABSTRACT. Massive lobar emphysema in the middle lobe of the right lung was observed in a dog brought to our clinic with sudden onset of tension pneumothorax, and lobectomy was performed to excise it. Pathological examination resulted in a diagnosis of congenital bronchiectasis associated with bronchial cartilage hypoplasia. Two cases of diagnosis and successful treatment of congenital lobar emphysema have been reported in dogs.

KEY WORDS: bronchiectasis, canine, congenital lobar emphysema.

In small animals, there have been very few reports about congenital anomalies of the lower respiratory tract, especially lung, because of their early death after birth. As far as the author's knowledge, only two diagnosed and one suspected cases of the congenital lobar emphysema have been reported in $\operatorname{dogs}[1,2]$. The principal clinical sign is progressive dyspnea, followed by coughing. In addition, exercise intolerance, vomiting, tachypnea, cyanosis and loud respiratory sounds may be associated [2]. In this note, it is reported that the dog with emphysema in the right middle lobe was recovered by lobectomy, and diagnosed as congenital bronchiectasia with hypoplasia of the bronchial cartilage.

The dog was a 22-month-old male Pomeranian weighing $4.0 \mathrm{~kg}$ and was brought to the clinic because of the sudden onset of coughing, loss of vigor, and loss of appetite the day before. Similar manifestations had occurred one year previously, but the dog had recovered without treatment.

At the time of the initial physical examination, the dog's body temperature was $38.7^{\circ} \mathrm{C}$, the lung sounds were prominent and coarse, the heart sounds were indistinct, and labored breathing was noted at rest. The color of the visible mucous membranes was normal. There were no major abnormal findings among the results of the blood tests. Plain x-ray films of the chest confirmed the retention of a large volume of air in the right pleural cavity, and a shadow that seemed to represent an atrophied right middle lobe was observed (Fig. 1). The dog was hospitalized and placed in an oxygen room as an emergency measure. A right thoracentesis was performed under sedation, and a large volume of air was removed. Afterwards, we performed a series of $\mathrm{x}-$ ray studies while keeping the dog in rest.

Because a huge bulla that seemed to be located in the position of the right middle lobe was first noted after the air was removed and was found to be tending to become more distinct and larger (Fig. 2).
On hospital day 5 lobectomy was performed through a thoracotomy at intercostal spaces 5-6 on the right side.

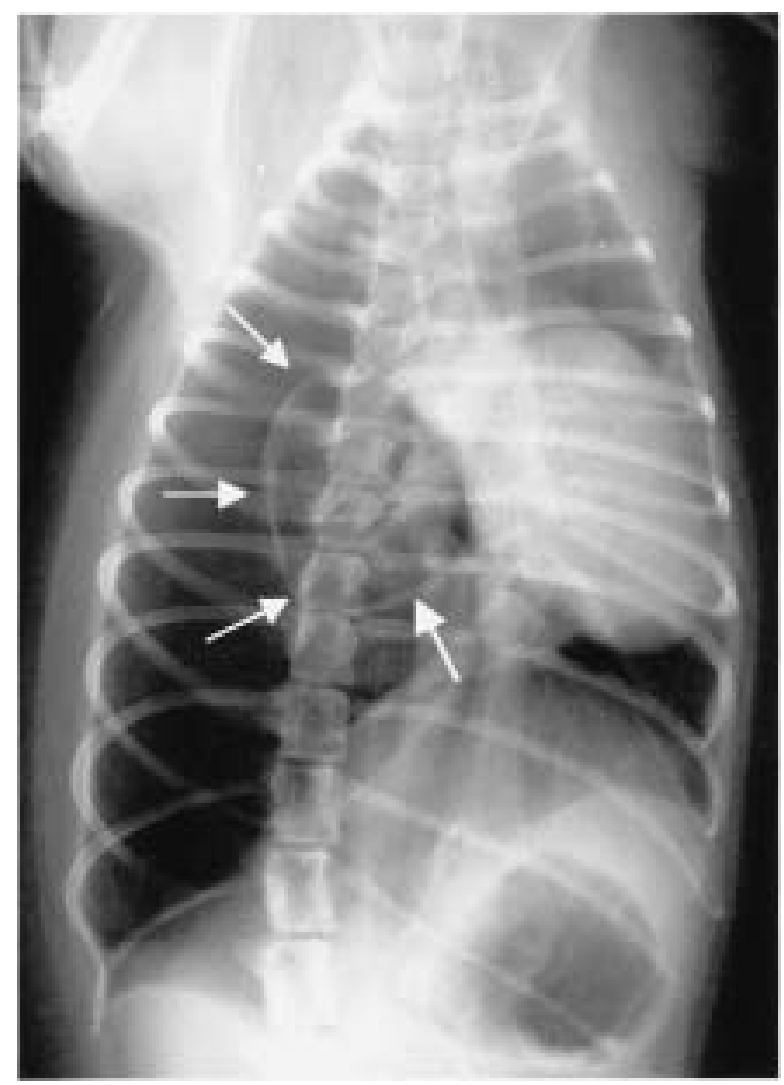

Fig.1. Dorso-ventral view plain x-ray film of the thorax at the time of the initial examination. Pneumothorax is seen in the right pleural cavity, the heart is compressed toward the left thoracic wall, and a slightly emphysematous right middle lobe (arrows) was observed. 


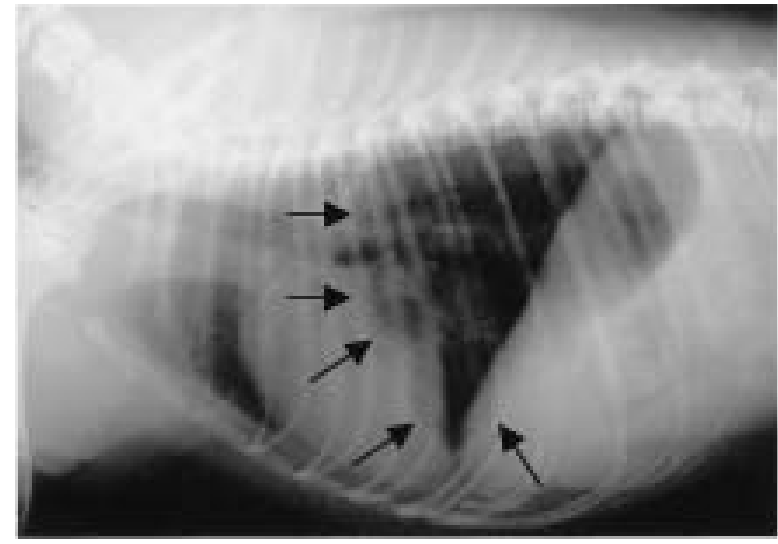

Fig.2. Right lateral view plain x-ray film before surgery. A large emphysematous lobe (arrows) of the lung extending from the fifth to the twelfth thoracic segment is observed.

Anesthesia consisted of premedication with atropine sulfate and acepromazine maleate, followed by induction with intravenous ketamine hydrochloride and diazepam, endotracheal intubation, and maintenance by inhalation anesthesia with a mixture of isoflurane and oxygen. Controlled respiration during the thoracotomy was achieved by intermittent administration of suxamethonium chloride. Intraoperative exploration revealed ballooning and thinning of the entire right middle lobe, and only scattered areas of what appeared to be normal lung were seen at the margin of the lung. Grossly the right middle lobe had the external appearance of the "vanishing lung" observed in lobe containing advanced emphysematous cysts (Fig. 3). The right middle lobe was excised, and the chest was closed in the routine manner. The postoperative course was smooth, and the dog was discharged on postoperative day 7. Follow-up examinations have been performed over a 15-month period, and no particularly abnormal findings have been detected thus far.

The pathological examination revealed the formation of a large cystic lesion within the lung that communicated with the main bronchus. The cyst wall was composed of fibrous connective tissue. An extensive area of the cyst cavity was not lined by epithelium, and granulation tissue associated with old changes was also seen. Islands of lung tissue persisted at the periphery of the cyst. The lung tissue was hyperemic, and a pathological picture of pneumonia was seen in scattered areas at the margin. Since an area where part of the cyst wall communicated with pulmonary alveolar tissue was confirmed and bronchial epithelium possessing villi was seen in the communication between the cyst and the bronchus, a diagnosis of congenital bronchiectasia associated with bronchial cartilage hypoplasia was made (Fig. 4).

Congenital lobar emphysema in the dog was first reported by Herrtage and Clarke, who found emphysematous bullae at the postmorten examination [3]. Bronchial cartilage dysplasia [2, 4, 7], hypoplasia [1, 6], and aplasia [8] were noted in later reports, and we observed bronchial hypoplasia in our own case. The same as Karnak et al. pointed out in

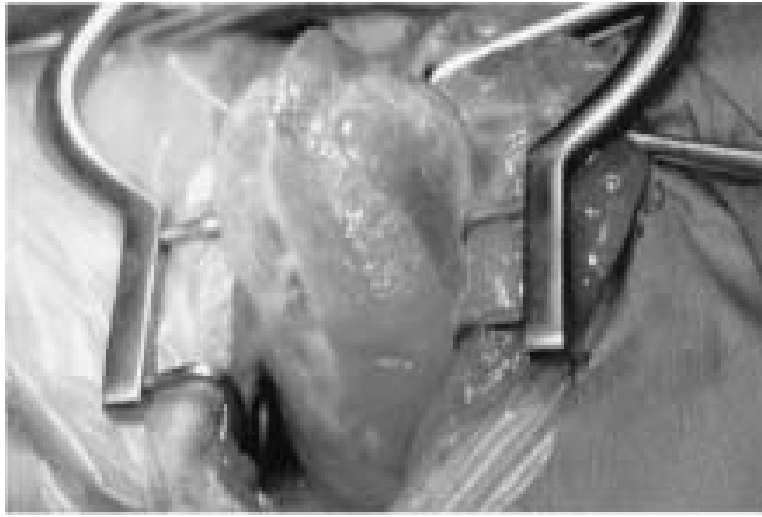

Fig.3. Emphysematous right middle lobe during surgery. Only a slight amount of normal appearing lung is visible at the margin of the lobe, and the whole lobe has been stretched out like a balloon.

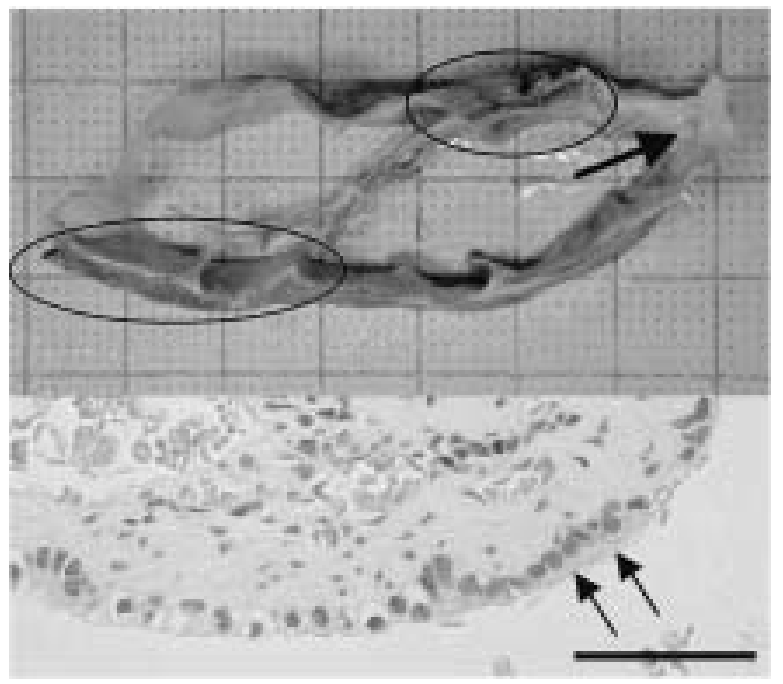

Fig.4. Pathological findings. There was a cystic lesion that communicated with the main bronchus (large arrow), and islands of lung tissue (circles) remained at the edge of the cyst. Bronchial epithelium (small arrows) was seen in the communication between the cyst and the bronchus. Hematoxylin and eosin stain. Bar=50 $\mu \mathrm{m}$.

humans [5], there appears to be a close relationship between lobar emphysema and bronchial cartilage abnormalities in the dog too.

Based on the total of four cases reported thus far, consisting of two cases of congenital lobar emphysema [1, 2], one likely case [6], and our own case, surgery seems to have been the most common form of treatment for emphysema in a single relatively small lobe, because the lesions were limited to the right middle lobe in three out of the four cases and in the other it was limited to the left cranial lobe. If the emphysema is multifocal and/or in a large lobe, it is important to determine the suitability of surgery and to make a 
prognosis. Computed tomography, bronchoscopy, and scintigraphy are needed as well as plain thoracic radiographs to make more detailed morphological evaluations for this purpose. Echocardiography, blood gas analysis, angiocardiography, esophagography, and spirometry should also be performed to exclude other diagnoses. There were no visible abnormalities in any of the lobes in the right pleural cavity in our case, but follow-up is needed, because the left lung and pulmonary parenchyma could not sufficiently examined during surgery.

The diagnosis and treatment of lobar emphysema were relatively easy because of the spontaneous pneumothorax, although no cases complicated by pneumothorax had ever been cured.

\section{REFERENCES}

1. Amis, T.C., Hager, D., Dungworth, D.L. and Hornof, W. 1987. J. Am. Anim. Hosp. Assoc. 23: 321-329.

2. Billet, J-P. H. G. and Sharpe, A. 2002. J. Small Anim. Pract, 43: $84-87$.

3. Herrtage, M.E. and Clarke, D.D. 1985. J. Small Anim. Pract. 26: $453-464$

4. Hoover, J.P., Henry, G.A. and Panciera, R.J. 1992. J. Am. Vet. Med. Assoc. 201: 599-602.

5. Karnak, I., Senocak, M.E., Ciftci, A.O. and Buyukpamukcu, N. 1999. J. Pediatr. Surg. 34: 1347-1351.

6. Orima, H., Fujita, M., Aoki, S., Washizu, M., Yamagami, T., Umeda, M. and Sugiyama, M. 1992. J. Vet. Med. Sci. 54: 797798.

7. Tennant, B.J. and Haywood, S. 1987. J. Small Anim. Pract. 28: 109-116.

8. Voorhout, G., Goedegebure, S.A. and Nap, R.C. 1986. Vet. Pathol. 23: 83-84. 